- 研究报告・

\title{
红外相机技术与其他几种森林鸟类 多样性调查方法的比较
}

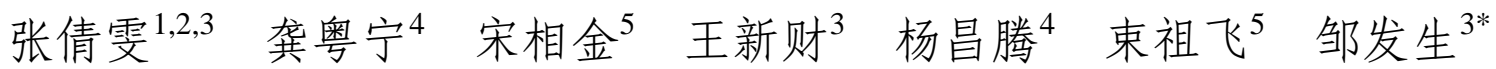 \\ 1 (中国科学院华南植物园, 广州 510650) \\ 2 (中国科学院大学, 北京 100049) \\ 3 (广东省生物资源应用研究所, 广东省动物保护与资源利用重点实验室, 广东省野生动物保护与利用公共实验室, 广州 510260) \\ 4 (广东南岭国家级自然保护区管理局, 广东乳源 512727) \\ 5 (广东车八岭国家级自然保护区管理局, 广东始兴 512528)
}

摘要: 为了探讨红外相机技术、网捕法、固定距离样线法和固定半径样点法对森林鸟类多样性调查的有效性和实 用性, 我们于2011-2016年, 用这几种方法调查了广东南岭国家级自然保护区和车八岭国家级自然保护区的鸟类 多样性。在南岭, 固定距离样线法和固定半径样点法记录鸟类222种, 网捕鸟类43种, 红外相机拍到鸟类47种; 其 中1种鸟仅网捕到, 6种鸟仅被红外相机拍到, 164种鸟仅被固定距离样线法和固定半径样点法记录到。在车八岭, 固 定距离样线法和固定半径样点法记录鸟类109种, 网捕鸟类42种, 红外相机拍到鸟类27种, 其中9种鸟仅网捕到, 3 种鸟仅被红外相机拍到, 97种鸟仅被固定距离样线法和固定半径样点法记录到。随着鸟类的体重、体长、翅长和 跗踮增加, 网捕到的鸟类个体数量减少, 鸟类身体大小与网捕到的鸟类数量呈显著负相关; 而红外相机拍到的鸟 类数量随鸟类的身体大小增大而增加, 并且呈显著的正相关。本文的结果显示, 网捕法和红外相机技术针对不同 类群的鸟类调查效力不同, 但都是固定距离样线法和固定半径样点法的有效补充。建议今后开展森林鸟类多样性 调查与监测时, 尽量采用多种研究方法相结合, 以求达到最优的效果。

关键词: 鸟类多样性; 红外相机技术; 网捕法; 固定距离样线法; 固定半径样点法; 生物多样性监测

\section{Comparing the effectiveness of camera trapping to traditional methods for biodiversity surveys of forest birds}

Qianwen Zhang ${ }^{1,2,3}$, Yuening Gong ${ }^{4}$, Xiangjin Song ${ }^{5}$, Xincai Wang ${ }^{3}$, Changteng Yang ${ }^{4}$, Zufei Shu ${ }^{5}$, Fasheng Zou ${ }^{3 *}$

1 South China Botanical Garden, Chinese Academy of Sciences, Guangzhou 510650

2 University of Chinese Academy of Sciences, Beijing 100049

3 Guangdong Key Laboratory of Animal Conservation and Resource Utilization, Guangdong Public Laboratory of Wild

Animal Conservation and Utilization, Guangdong Institute of Applied Biological Resources, Guangzhou 510260

4 Administration of Guangdong Nanling National Nature Reserve, Ruyuan, Guangdong 512727

5 Administration of Guangdong Chebaling National Nature Reserve, Shixing, Guangdong 512528

\begin{abstract}
To compare the effectiveness of camera trapping to more common mist netting, fixed-distance line transect, and fixed-radius point count methods of forest birds, we surveyed bird species diversity at Nanling National Nature Reserve and Chebaling National Nature Reserve, Guangdong, China from 2011 to 2016. At Nanling, 222 bird species were recorded by fixed-distance line transect and fixed-radius point count methods, 43 bird species were captured by mist netting and 47 bird species were trapped by cameras. One species was captured only by mist netting, six species only by camera-trapping, and 164 species only by fixed-distance line transect and fixed-radius point count methods. At Chebaling, 109 bird species were recorded by fixed-distance line transect and fixed-radius point count methods, 42 by mist nets and 27 by cameras. Nine
\end{abstract}


species were recorded only by mist netting, three only by camera-trapping, and 97 only by fixed-distance line transect and fixed-radius point count methods. At both sites, the number of birds captured by mist netting was negatively correlated with increasing body size, whether measured as body weight, body length, wing length, or tarsus length. By contrast, the number of birds photographed by camera traps was positively correlated with the same measures of body size. These results show that bird surveys using different methods may yield different results and that method should be selected to suit the body size of the target bird species. In surveying bird diversity of a wide range of body sizes, mist netting and camera trapping together would be complimentary methods. Our results suggest that a combination of research methods may be necessary to obtain reliable avian diversity estimates.

Key words: avian diversity; camera trapping; mist netting; fixed-distance line transect; fixed-radius point count; biodiversity monitoring

传统的鸟类多样性调查有多种方法, 包括标图 法(spot-map census method)、样线法(transect census method)、样点法(point count method)等(Verner \& Ritter, 1985; Bibby et al, 2000; 许龙等, 2003)。标图 法是将一定地区观察到的每只鸟的位点标记在已 知比例的方格地图上, 通过图形转换, 确定位点群, 计算密度等来调查鸟类群落(郑光美, 2012)。标图 法 需要花费大量的时间和资金, 效率较低, 目前只在 欧美少数国家得以应用(Bibby et al, 2000; Gregory, 2000)。样点法和样线法是目前鸟类调查中最常见的 方法, 由于其数据收集的高效性和灵活性, 这两种 方法特别适用于进行鸟类监测(Gibbons \& Gregory, 2006; 吴飞和杨晓君, 2008; 郑光美, 2012)。但多数 森林林下幽暗, 对行为隐蔽、少鸣唱的鸟类来说, 仅采用需要靠视觉和听觉的样线法和样点法进行 调查, 往往调查到的种类不完全。网捕法(mist net) 对林下活动、性情隐蔽的鸟种调查特别有效, 并且 可以准确鉴定鸟种, 进行环志, 检查鸟类换羽状况 等, 多数鸟类研究者把它作为样线法和样点法的补 充(邹发生和陈桂珠, 2003; Dunn \& Ralph, 2004), 并 且已经在海南、广东、贵州等地开展了应用(邹发生 和陈桂珠, 2004; 邹发生和杨琼芳, 2005; 罗祖奎等, 2013)。然而不论在哪个季节, 网捕法都很难捕到某 些鸟种, 如空中受食的燕、猛禽等(Wang \& Finch, 2002)。一般认为网捕法仅对活动于森林 $4 \mathrm{~m}$ 以下的 鸟类比较有效, 对于高于 $4 \mathrm{~m}$ 活动的鸟类效果较不 明显(Zou et al, 2010)。对于大型鸟类而言, 网捕法 也无法有效调查其多样性, 因为大型鸟类有较大的 足部和翅膀, 容易在网中跳动并逃脱(Jenni et al, 1996; Steenweg et al, 2017)。

相比传统方法, 红外相机技术具有众多优势:
可以持续工作，不易受天气和地形等环境因子的影 响, 可节省资金和人力, 减少对动物的干扰, 尤其 对于夜行性和行为隐蔽种的调查优势明显(O’Connell et al, 2011; 李晟等, 2014; 肖治术等, 2014b), 因此该方法逐渐被应用到生物监测和研究中。早在 19世纪末, 国外就有学者利用相机进行野生动物调 查(Wallihan \& Wallihan, 1906), 当时主要用于调查 大型兽类, 如马鹿(Cervus elaphus)、骡鹿(Odocoileus hemionus)、叉角羚(Antilocapra americana)、美 洲狮(Felis concolor)、猞猁(Lynx rufus)等。Pearson (1959)开发了触发式红外相机后, 在野外拍到了兽 类、鸟类、甚至爬行类，但是主要还是应用于兽类 研究。国内对于红外相机的应用起步相对较晚, 20 世纪末, Pei (1995)在台湾地区、马世来和 Harris (1996)在大陆首次应用红外相机进行野外生物调查; 21世纪初, 卢学理等(2005)、马鸣等(2006)利用红外 相机对大熊猫(Ailuropoda melanoleuca)、雪豹(Panthera uncia) 等兽类进行了研究。随着红外相机技术 的不断成熟, 其应用不再局限于兽类野外调查, 如 Chan等(2005)使用红外相机调查了霸王岭保护区的 鸟类区系, 发现了不少珍稀濒危鸟类和保护区新记 录种。近年来，该技术的应用范围仍在不断扩大， 越来越多地被用于调查和监测鸟类多样性(Dinata et al, 2008; 斯幸峰和丁平, 2014; 肖治术等, 2014a; Burton et al, 2015; 朱淑怡等, 2017)。

本文比较了几种调查森林鸟类多样性方法的 效果, 同时研究鸟类的体重和体形是否影响鸟类的 网捕或者红外相机技术拍摄效果, 期望从中得到一 些启示, 倡导在鸟类多样性调查与研究上, 充分利 用不同的研究手段和方法, 以达到更好的调查和监 测效果。 


\section{研究方法}

\section{1 研究地区概况}

研究在广东南岭国家级自然保护区(下文简称 南岭)和广东车八岭国家级自然保护区(下文简称车 八岭)进行。

南岭位于广东省北部, 地理坐标为 $112^{\circ} 41^{\prime}-$ $113^{\circ} 15^{\prime} \mathrm{E}, 24^{\circ} 39^{\prime}-28^{\circ} 08^{\prime} \mathrm{N}$, 总面积 $56,300 \mathrm{ha}$, 是目 前广东省面积最大的森林类型国家级自然保护区。 属于花岗岩山地, 海拔一般在 $1,000 \mathrm{~m}$ 以上, 超过 $1,200 \mathrm{~m}$ 的山峰有 85 座, 其中石坑崆为广东省最高 峰(海拔1,902 m)(庞雄飞, 2003)。保护区位于南亚热 带到中亚热带的过渡地带, 属于亚热带季风区。年 平均气温 $19.5-20.3^{\circ} \mathrm{C}$, 年平均降水量 $1,705 \mathrm{~mm}$, 并 且集中在3-8月(田怀珍和邢福武, 2008)。主要植被 类型为亚热带山地常绿阔叶林, 常见乔木树种有观 光木(Tsoongiodendron odorum)、浙江润楠(Machilus chekiangensis)、蜡瓣花(Corylopsis sinensis)、雷公青 冈(Cyclobalanopsis hui)、荺蒴(Castanopsis fissa)等; 灌木层常见的有天料木(Homalium cochinchinense)、 黄丹木姜子(Litsea elongata)、毛果巴豆(Croton lachnocarpus)等; 草本层以金毛狗(Cibotium barometz) 数量最多(王发国等, 2013)。

车八岭位于广东省韶关市始兴县东南部，地 理位置为 $114^{\circ} 09^{\prime} 04^{\prime \prime}-114^{\circ} 16^{\prime} 46^{\prime \prime}$ E, 244ㅇ' $29^{\prime \prime}-$ $24^{\circ} 46^{\prime} 21^{\prime \prime} \mathrm{N}$, 总面积 $7,545 \mathrm{ha}$ 。年平均气温 $19.6^{\circ} \mathrm{C}$, 年平均降水量 $1,468 \mathrm{~mm}$, 主要保护对象是中亚热带 常绿阔叶林及珍稀动植物, 常见乔木有枫香(Liquidambar formosana)、木荷 (Schima superba)、深山 含 笑(Michelia maudiae)、金叶含笑(M. foveolata)等; 灌 木层常见的有毛冬青(Ilex pubescens)、大罗伞(Ardisia hanceana)、木姜子(Litsea pungens)等。草本层 常见的有草珊瑚(Sarcandra glabra)、耳草(Hedyotis auricularia)等(陈涛等, 1992)。

\section{2 固定距离样线法和固定半径样点法}

根据南岭植被类型的空间分布格局以及海拔 梯度变化特征, 以分层抽样法设计 17 条观测样线, 即从海拔200-1,923 m, 海拔每升高100 m设置1条 样线。车八岭设置10条样线。鸟类抽样调查采用固 定距离样线法和固定半径样点法同时进行, 这两种 互补结合的方法被认为是最为高效的森林鸟类群 落调查方法(Bibby et al, 2000)。采用固定距离样线
法调查时, 每条样线长约2.5-3.5 km, 两名调查人 员同时沿固定样线以 $1-2 \mathrm{~km} / \mathrm{h}$ 的速度行进，用 $10 \times$ 42 Olympus双筒望远镜记录样线两侧各 $50 \mathrm{~m}$ 距离 内发现的鸟类个体(包括听到和见到的); 固定半径 样点法是在样线上每隔200 m设1处固定样点、每条 样线上设 10 个样点，同时用已标号的样点牌作为标 志挂在树上，并用GPS定好坐标，方便下次定点重 复调查。调查人员在到达样点后立即开始记录样点 周围50 m内听到和见到的鸟类, 每个样点的计数时 间为 $10 \mathrm{~min}$ 。记录鸟类的种类、数量、到样线的垂 直距离或者到样点圆心的距离。南岭的调查时间为 2011-2016年，共6年; 车八岭的调查时间为20152016年，共2年。每年的春、夏、秋、冬各调查1次。

\section{3 红外相机设置}

根据保护区工作人员的介绍，选择人为活动 少、鸟类种类丰富的山路(样线)安放红外相机 (Rowcliffe et al, 2014)。相机安装在树干上，离地大 约50 cm, 详细安装方法见蔡玉生等(2016)。相机型 号为猎科Ltl 5210数码红外相机，每次触发后拍照3 张，再录制 $30 \mathrm{~s}$ 的视频，触发间隔为 $1 \mathrm{~s}$ 。南岭安放 红外相机时间为2011年1月1日至2016年1月 8日， 全部安放在 $112^{\circ} 42^{\prime} 45^{\prime \prime}-113^{\circ} 04^{\prime} 08^{\prime \prime}$ E，2441 ${ }^{\prime} 01^{\prime \prime}-$

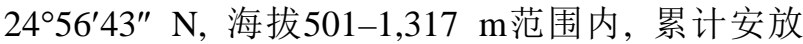
124 台; 车八岭安放相机时间为 2011 年 1 月 3 日至 2016 年 11 月 1 日，全部相机安放在 $114^{\circ} 09^{\prime} 57^{\prime \prime}$ $114^{\circ} 16^{\prime} 42^{\prime \prime}$ E， $24^{\circ} 42^{\prime} 45^{\prime \prime}-24^{\circ} 44^{\prime} 11^{\prime \prime} \mathrm{N}$ ，海拔 372 $1,070 \mathrm{~m}$ 范围内，累计安放66台。

\section{4 网捕法}

使用全国鸟类环志中心推荐的雾网，长12 m, 高 $2.6 \mathrm{~m}$, 网眼大小 $36 \mathrm{~mm}$ 。选择林间开阔的小路张 网, 张网时2-3个网首尾相连, 网的下纲垂到地面 或离地 $0.1 \mathrm{~m}$ 高, 网长轴方向随地势而定, 各网不尽 相同(邹发生和陈桂珠, 2003, 2004)。张网时间一般 在6:30-18:00 (从开第1张网到收最后1张网), 下雨 天不张网。每隔2-3 hi《网1次，捕到鸟后迅速将鸟 取下, 记录鸟的种类、数量、上网时间、上网高度 等信息, 最后将鸟在原地释放。野外张网工作从 2012年7月至2016年12月，每个保护区每次调查开 10 张网，其中南岭共张网8次、每次 5-7天; 车八岭 共张网24次、每次3-4天。每年布网的地点基本保 持不变, 南岭张网地点坐标为 $113^{\circ} 0^{\prime} 12^{\prime \prime}-113^{\circ} 0^{\prime} 22^{\prime \prime}$

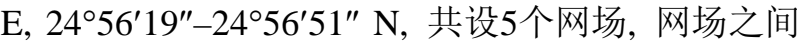


相隔 200-300 m; 车八岭张网地点的中心位置是 $114^{\circ} 15^{\prime} 02^{\prime \prime} \mathrm{E}, 24^{\circ} 43^{\prime} 07^{\prime \prime} \mathrm{N}$, 也设 5 个网场，网场之间 相隔200-300 m。显然, 不论是在南岭还是在车八 岭, 张网的范围完全在安放红外相机的范围内。

\section{5 数据处理}

鸟类身体数据(体重、体长、翅长、跗蹠长)一 方面来自多年网捕到鸟的测量数据, 另一方面来自 于文献(赵正阶, 2001a, b; Dunning, 2007)。记录红外 相机拍摄鸟类照片的种类和日期。对于同一相机在 同一地点拍摄的照片，时间间隔小于 $30 \mathrm{~min}$ 、同一 种鸟的连续照片称为 1 张有效照片。本文将红外相 机拍摄有效照片数量占总数量 $5 \%$ 以上的种作为优 势种, 其余 3 种方法均以记录到的鸟类数量占总数 量 $5 \%$ 以上的种作为优势种。将红外相机拍到鸟类数 量和网捕鸟类数量进行 $\log (\mathrm{N}+1)$ 转换, 身体数据 (体重、体长、翅长、跗蹠长) 进行 $\log$ 转换后再进行 相关分析和作图, 红外相机拍到的鸟类数量和网 捕鸟类数量与鸟类身体大小的相关性分析采用
Spearman相关性系数 $(r)$, 显著性水平 (双尾检验) 设 为: $\alpha=0.05$ 。统计分析软件为SPSS 20, 作图软件为 SigmaPlot 12.5。

\section{结果}

\section{1 物种多样性}

在南岭, 固定距离样线法和固定半径样点法记 录鸟类222种, 优势种为灰眶雀鸤 (Alcippe morrisonia)和栗耳倜(Yuhina castaniceps), 它们分别占 总记录个体数的 $22.95 \%$ 及 $9.91 \%$ 。网捕鸟类 43 种, 优 势种是灰眀雀鹤和红嘴相思鸟(Leiothrix lutea), 分 别占总网捕数量的 $34.8 \%$ 和 $13.3 \%$ 。南岭累计拍摄 33,408 相机日，获取鸟类有效照片 3,421 张，拍到鸟 类47种, 优势种是白鹇(Lophura nycthemera)、紫啸 冻(Myophonus caeruleus)、白眉山麅鸪(Arborophila gingica)、黑领噪㓔(Garrulax pectoralis)和黄腹角雉 (Tragopan caboti), 分别占该地区红外相机拍到鸟 类总数量的 $36.3 \% 、 21.5 \% 、 9.5 \% 、 7.6 \%$ 和 $5.3 \%$ 。四 种方法共记录到鸟类229种, 其中棕腹大仙能(Niltava davidi)仅网捕到; 丘劀(Scolopax rusticola)、绿 翅金鸭 (Chalcophaps indica)、橙头地鸫(Zoothera citrina)、白眉地冻 (Z. sibirica)、白眉冻 (Turdus obscurus)、黑喉噪鹋(Garrulax chinensis) 6种鸟仅被 红外相机拍到; 164种鸟仅被固定距离样线法和固
定半径样点法记录到。网捕法和红外相机法记录的 鸟类名录见附录 1 。

在车八岭, 固定距离样线法和固定半径样点法 记录鸟类109种, 优势种为黑短脚䍩 (Hypsipetes leu-

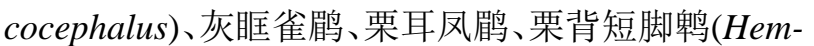
ixos castanonotus)和灰树鹊(Dendrocitta formosae), 分别占总记录个体数的 $22.51 \% 、 14.64 \% 、 7.41 \%$ 、 $5.83 \%$ 和 $5.05 \%$ 。网捕鸟类 42 种，优势种是灰眀雀鹛、 灰树鹊、灰背冻(Turdus hortulorum)、红嘴相思鸟和 栗背短脚鹐，分别占总网捕数量的 $36.0 \% 、 11.4 \%$ 、 9.5\%、6.5\%和 $5.6 \%$ 。车八岭累计拍摄 7,039 相机日， 获取鸟类有效照片2,164张，拍到鸟类27种，优势种 是白酠和黑领噪鹛, 分别占该地区拍到鸟类总数量 的 $49.4 \%$ 和 $32.5 \%$ 。四种方法共记录到鸟类 121 种，其 中日本松雀鹰(Accipiter gularis)、红翅风头鹃(Clamator coromandus)、栗啄木鸟(Celeus brachyurus)、 斑姬啄木鸟(Picumnus innominatus)、红尾歌鸲(Luscinia sibilans)、日本歌鸲(Erithacus akahige)、灰纹 㫽(Muscicapa griseisticta)、淡脚柳莺(Phylloscopus tenellipes)和黄眉鸥(Emberiza chrysophrys) 9种鸟仅 网捕到; 丘憰、白眉冻和黑喉噪鿬3种鸟仅被红外相 机拍到; 97种鸟仅被固定距离样线法和固定半径样 点法记录到。车八岭保护区的网捕法和红外相机法 记录的鸟类名录见附录 1 。

\section{2 红外相机技术和网捕法记录鸟类数量与鸟类} 身体大小的关系

在南岭用红外相机拍到的鸟类中, 体重最小的 是红胁蓝尾鸲(Tarsiger cyanurus)和栗耳凤鸤, 最大 的是白鹇; 体长最小的是小燕尾(Enicurus scouleri), 最大的是白洅；翅长最小的是褐顶雀鸤 (Alcippe brunnea), 最大的是白洅鸟; 跗蹠长最小的是普通夜 鹰(Caprimulgus indicus), 最大的是白泪鸟。车八岭红 外相机拍到的鸟类中, 体重最小的是红胁蓝尾鸲, 最大的是白酠; 体长最小的是灰眭雀鹛, 最大的是 白酠; 翅长最小的是灰眭雀鸤, 最大的是白酠; 跗 蹠长最小的是栗背短脚軠, 最大的是白酠。南岭和 车八岭红外相机拍到鸟类的数量与鸟体大小均呈 显著正相关(图1，图2，表1)。

在南岭网捕鸟类中，体重最小的是黄腰柳茑 (Phylloscopus proregulus), 最大的是斑头鹤鹠 (Glaucidium cuculoides); 体长最小的是栗头暡蒀 (Seicercus castaniceps), 最大的是红头咬鹃 

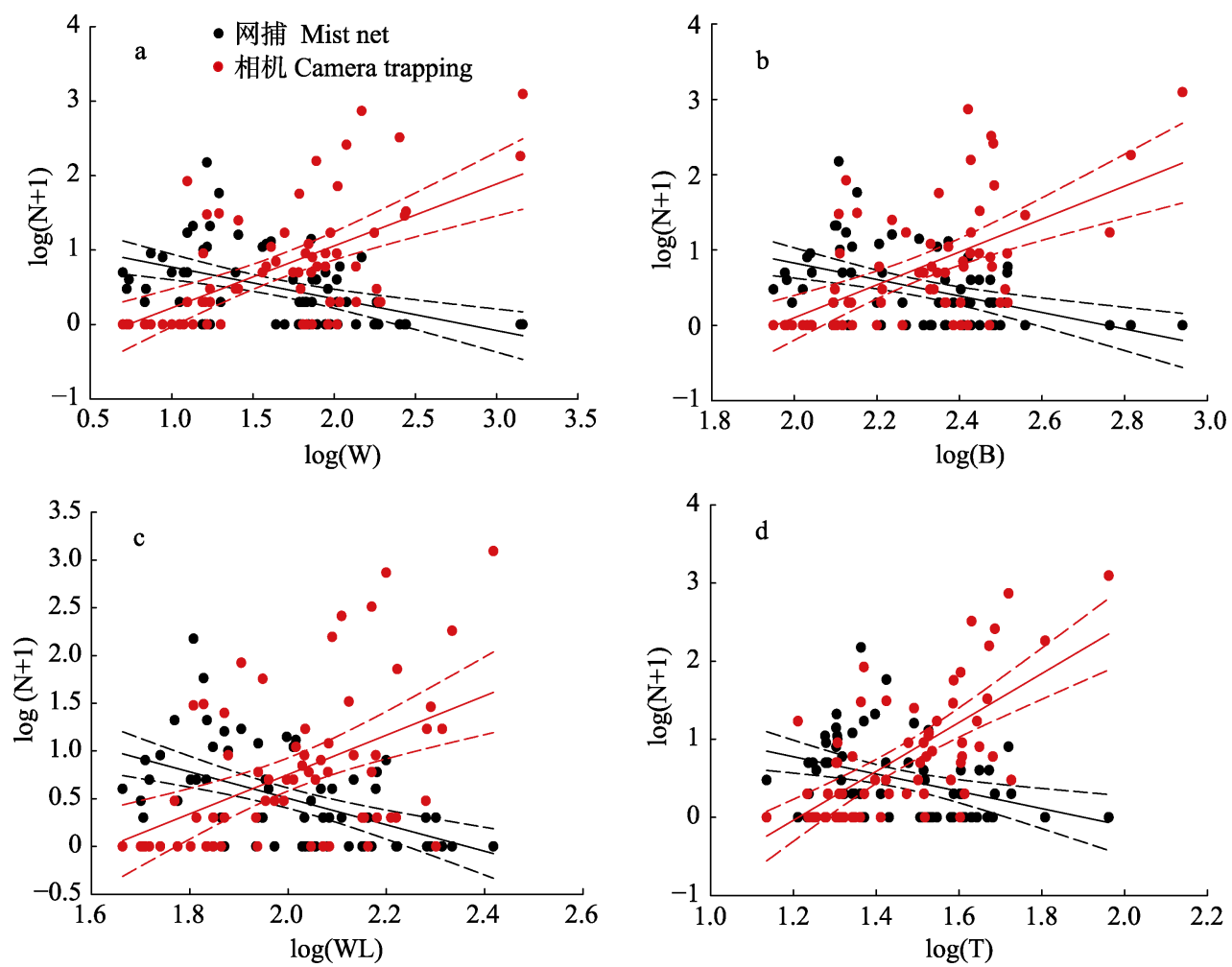

图1 南岭红外相机拍到和网捕鸟类数量与体重(a)、体长(b)、翅长(c)、跗蹠长(d)关系图。 $\mathrm{N}:$ 数量; W: 体重; B: 体长; WL: 翅长; $\mathrm{T}$ : 跗蹠长。虚线一 $95 \%$ 置信区间, 实线一拟合趋势线。

Fig.1 Relationship between bird number by camera trapping or mist net and bird body weight (a), body length (b), wing length (c), tarsus length (d) at Nanling, respectively. N, Number; W, weight; B, Body length; WL, Wing length; T, Tarsus length. Dotted line, 95\% confidence interval; Solid line, curve fit.

(Harpactes erythrocephalus); 翅长最小的是金色鸦 雀(Paradoxornis verreauxi), 最大的是中杜鹃 $(\mathrm{Cu}-$ culus saturatus); 跗蹠长最小的是栗头鹃荣, 最大 的是松雀鹰(Accipiter virgatus)。车八岭网捕鸟类中, 体重最小的是黄腰柳蒀, 最大的是斑头鹰鹠; 体长 最小的黄腰柳莺, 最大的是红头咬鹃; 翅长最小的 是叉尾太阳鸟(Aethopyga christinae), 最大的是虎 斑地冻(Zoothera dauma); 跗蹠长最小的是斑姬啄 木鸟, 最大的是日本松雀鹰。南岭和车八岭网捕鸟类 的数量与鸟体大小均呈显著负相关(图1，图2，表1)。

\section{3 讨论}

在鸟类多样性调查中, 样线法(周放, 1986)、样 方法(丁平等, 1989)、样点法(Zou et al, 2012)等传统 方法发挥了重要作用，但受调查强度、时间和方法 本身的限制, 均无法完整记录鸟类多样性的状况。 邹发生(2002)在海南尖峰岭和张强(2011)在广东鼎 湖山已结合网捕法和样点法进行森林鸟类的研究, 但是到目前为止，还没有结合样线法或者样点法、
网捕法和红外相机监测鸟类多样性的报道。本文第 一次应用 4 种方法开展森林鸟类多样性调查, 并比 较了各种方法的特点。

网捕法是鸟类监测的一种重要方法, 对研究鸟 类组成、种群大小等十分有用(Levey, 1988), 然而该 方法在调查鸟类多样性时也有一些缺点，易受外部 环境, 如栖息地类型(Rappole et al, 1998)、网眼大小 (Piratelli, 2003)、鸟的飞行高度(Whitman et al, 1997) 的影响。Zou等(2010)在海南岛尖峰岭的研究表明, 网捕法一般对在 $4 \mathrm{~m}$ 以下活动的鸟类非常有效，但 也有一些在林冠或者亚冠层活动的鸟类被捕到, 如 多在 8-9 $\mathrm{m}$ 和 11-13 $\mathrm{m}$ 高度活动的白喉冠軠 (Alophoixus pallidus)和银胸丝冠鸟(Serilophus lunatus)。本研究也捕到常在林冠层活动的赤红山椒 鸟(Pericrocotus flammeus)和灰喉山椒鸟(P. solaris) 等。森林中的光线甚至也对网捕产生影响，一般在 6,000 lux至8,000 lux条件下，会有更多的鸟被捕获 (Zou et al, 2010)。本研究在两个保护区共有10种鸟 仅被网捕到, 说明网捕法确是样线法和样点法的有 

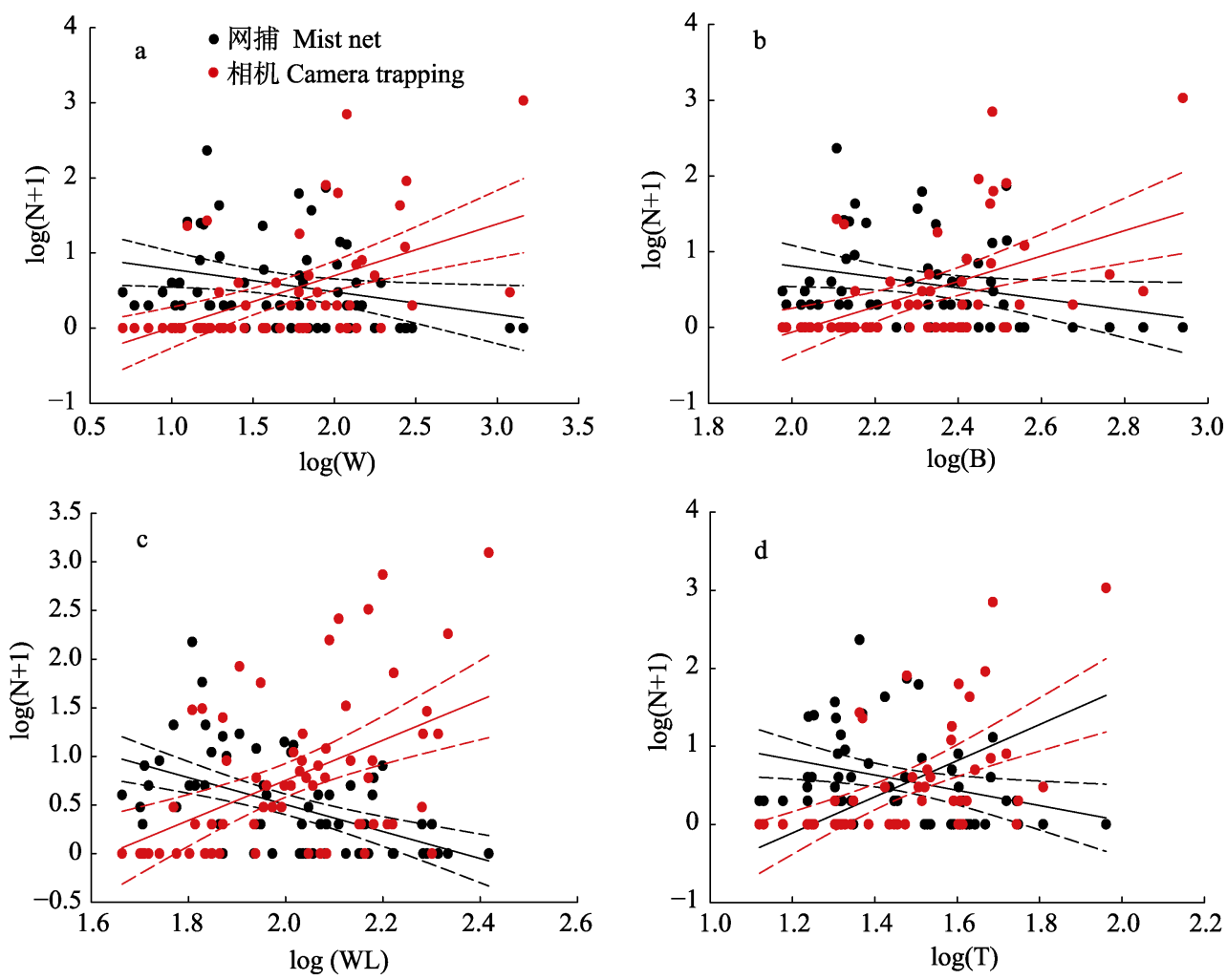

图2 车八岭红外相机拍到和网捕鸟类数量与体重(a)、体长 (b)、翅 (c)、跗蹠长(d)关系图。 $\mathrm{N}$ : 数量; W: 体重; B: 体长; WL: 翅长; $\mathrm{T}$ : 跗蹠长。虚线一 $95 \%$ 置信区间, 实线一拟合趋势线。

Fig. 2 Relationship between bird number by camera trapping or mist net and bird body weight (a), body length (b), wing length (c), tarsus length (d) at Chebaling, respectively. N, number; W, Weight; B, Body length; WL, Wing length; T, Tarsus length. Dotted line, 95\% confidence interval; Solid line, curve fit.

表1 红外相机拍到鸟类和网捕鸟类数量与鸟类身体大小的相关性

Table 1 The correlations between capture number derived from camera trapping and mist netting data and bird body size

\begin{tabular}{|c|c|c|c|c|}
\hline & 体重 Weight & 体长 Body length & 翅长 Wing length & 跗蹠长 Tarsus length \\
\hline \multicolumn{5}{|l|}{ 南岭 Nanling } \\
\hline 相机 Camera trapping & $r=0.525, \operatorname{sig}<0.001$ & $r=0.547$, sig $<0.001$ & $r=0.462, \operatorname{sig}<0.001$ & $r=0.646, \operatorname{sig}<0.001$ \\
\hline 网捕 Mist net & $r=-0.504, \operatorname{sig}<0.001$ & $r=-0.443, \operatorname{sig}<0.001$ & $r=-0.538, \operatorname{sig}<0.001$ & $r=-0.424, \operatorname{sig}<0.001$ \\
\hline \multicolumn{5}{|l|}{ 车八岭 Chebaling } \\
\hline 相机 Camera trapping & $r=0.550$, sig $<0.001$ & $r=0.517, \operatorname{sig}<0.001$ & $r=0.448, \operatorname{sig}=0.001$ & $r=0.664, \operatorname{sig}<0.001$ \\
\hline 网捕 Mist net & $r=-0.349$, sig $=0.008$ & $r=-0.292$, sig $=0.029$ & $r=-0.340$, sig $=0.01$ & $r=-0.388, \operatorname{sig}=0.003$ \\
\hline
\end{tabular}

效补充; 同时, 网捕鸟类以灰眶雀鹋和红嘴相思鸟 等小型鸟类为主, 大型雉科鸟类从未被网捕到。这 也证实了鸟类身体大小会对网捕效果产生影响。

红外相机具有连续 $24 \mathrm{~h}$ 监测的优点, 是森林鸟 类调查方法的重要补充。本研究有6种鸟仅被红外 相机拍到, 说明红外相机技术可以在鸟类多样性调 查中发挥一定作用。同时, 本研究表明, 红外相机 拍到的鸟类数量与鸟的身体大小呈显著正相关, 其 原因可能是因为体型太小的鸟不能及时触发相机, 或是运动速度太快, 相机无法捕捉到清晰、特征明
显的照片(O’Brien \& Kinnaird, 2008; Tobler et al, 2008; Meek et al, 2014)。朱淑怡等(2017)同样发现红 外相机记录到的鸟类物种中, 林下层及地面层活动 的鸟类为绝对优势类群。不仅如此, 红外相机拍摄 到的鸟类数量还与安放地点的地形和植被条件、红 外相机数量、安放时间长短、设定的拍照参数等因 素有关(Meek et al, 2014), 将红外相机安放在鸟类 经常活动的地点, 野外布放的红外相机数量越多、 相机在野外工作的时间越长, 拍到的鸟类种类和数 量越多(陈奕欣等, 2016; 高歌等, 2017)。多数使用 
红外相机监测鸟类多样性的研究, 调查到的鸟种数 量比较有限, 如广东鼎湖山、广西花坪拍摄到鸟类 有效照片数分别为 315 和 547 张, 记录到的鸟类分别 为13和19种(肖治术等, 2014a; 汪国海等, 2014)。尽 管红外相机法仍然存在技术上的不足，但是与传统 调查方法相比，红外相机的优点如持续性、非损伤 性、隐蔽性等仍然非常明显，因此已成为野外生物 调查中不可缺少的工具。

本文通过结合固定距离样线法、固定半径样点 法、网捕法和红外相机技术等几种森林鸟类调查方 法, 在广东南岭国家级自然保护区和广东车八岭国 家级自然保护区调查森林鸟类多样性, 得出不同的 调查方法对不同鸟类类群捕获效果不同，且有各自 的特点。因此, 如果要彻底调查清楚森林鸟类多样性, 仅用单一的调查方法是不够的，必须结合多种调查 方法、用多种手段和技术才能达到较理想的效果。

\section{致谢：感谢广东省生物资源应用研究所张礼标研究} 员对文章修改给予的帮助!

\section{参考文献}

Bibby CJ, Burgess DN, Hill DA (2000) Bird Census Techniques. Academic Press, London.

Burton AC, Neilson E, Moreira D, Ladle A, Steenweg R, Fisher JT, Bayne E, Boutin S (2015) Wildlife camera trapping: A review and recommendations for linking surveys to ecological processes. Journal of Applied Ecology, 52, 675685.

Cai YS, Gong YN, Lu XL, Xiao ZS, Wang XC (2016) Camera trap survey of mammal diversity in Nanling forests. Ecological Science, 35(2), 57-61. (in Chinese with English abstract) [蔡玉生, 龚粤宁, 卢学理, 肖治术, 王新财 (2016) 南岭森林哺乳动物多样性的红外相机监测. 生态 科学, 35(2), 57-61.]

Chan BPL, Lee KS, Zhang JF, Su WB (2005) Notable bird records from Bawangling National Nature Reserve, Hainan Island, China. Forktail, 21, 33-41.

Chen T, Miao SY, Liao WB, Su ZY, Tang SQ, Zhang HD (1992) The floristic study of spermatophyte from Chebaling Nature Reserve in Northern Guangdong. Ecological Science, 1, 1-28. (in Chinese with English abstract) [陈涛, 缪绅裕, 廖文波, 苏志尧, 唐绍清, 张宏达 (1992) 广东车八岭自 然保护区植物区系地理研究. 生态科学, 1, 1-28.]

Chen YX, Xiao ZS, Li M, Wang XW, He CX, He GP, Li HS, Shi SJ, Xiang ZF (2016) Preliminary survey for the biodiversity of mammal and bird using camera traps in the west of mid-section Mt. Gaoligong. Acta Theriologica Sinica, 36,
302-312. (in Chinese with English abstract) [陈奕欣, 肖治 术，李明，王新文，何臣相，何贵品，李海曙，施顺金，向 左甫 (2016) 利用红外相机对高黎贡山中段西坡兽类和 鸟类多样性初步调查. 兽类学报, 36, 302-312.]

Dinata Y, Nugroho A, Haidir IA, Linkie M (2008) Camera trapping rare and threatened avifauna in west-central Sumatra. Bird Conservation International, 18, 30-37.

Ding P, Zhuge Y, Jiang SR (1989) Community ecology of birds on area of Gutian Mountain, Zhejiang Province. Acta Ecologica Sinica, 9, 121-127. (in Chinese with English abstract) [丁平, 诸葛阳, 姜仕仁 (1989) 浙江古田山自然 保护区鸟类群落生态研究. 生态学报, 9, 121-127.]

Dunn EH, Ralph CJ (2004) The use of mist nets as a tool for bird population monitoring. Studies in Avian Biology, 29, $1-6$.

Dunning JB (2007) CRC Handbook of Avian Body Masses, 2nd edn. CRC Press, London.

Gao G, Wang B, He CX, Luo X (2017) Biodiversity of birds and mammals in alpine habitat of Mt. Gaoligong, Lushui County, Yunnan. Biodiversity Science, 25, 332-339. (in Chinese with English abstract) [高歌, 王斌, 何臣相, 罗旭 (2017) 云南沪水高黎贡山高山生境的鸟兽多样性. 生物 多样性, 25, 332-339.]

Gibbons DW, Gregory RD (2006) Point counts or point transects. In: Ecological Census Techniques (ed. Sutherland WJ), pp. 324-332. Cambridge University Press, Cambridge.

Gregory RD (2000) Development of breeding bird monitoring in the United Kingdom and adopting its principles elsewhere. The Ring, 22, 35-44.

Jenni L, Leuenberger M, Rampazzi F (1996) Capture efficiency of mist nets with comments on their role in the assessment of passerine habitat use. Journal of Field Ornithology, 67, 263-274.

Levey DJ (1988) Tropical wet forest treefall gaps and distributions of understory birds and plants. Ecology, 69, 10761089.

Li S, Wang DJ, Xiao ZS, Li XH, Wang TM, Feng LM, Wang Y (2014) Camera-trapping in wildlife research and conservation in China: Review and outlook. Biodiversity Science, 22, 685-695. (in Chinese with English abstract) [李晟, 王 大军, 肖治术, 李欣海, 王天明, 冯利民, 王云 (2014) 红外相机技术在我国野生动物研究与保护中的应用与前 景. 生物多样性, 22, 685-695.]

Lu XL, Jiang ZG, Tang JR, Wang XJ, Xiang DQ, Zhang JP (2005) Auto-trigger camera traps for studying giant panda and its sympatric wildlife species. Acta Zoologica Sinca, 51, 495-500. (in Chinese with English abstract) [卢学理, 蒋志 刚, 唐继荣, 王学杰, 向定乾, 张建平 (2005) 自动感应 照相系统在大熊猫以及同域分布的野生动物研究中的应 用. 动物学报, 51, 495-500.]

Luo ZK, Liu LP, Wang Y, Niu JY, Liu LB, Li L (2013) Characteristics of the avian communities at Mt. Yuntaishan of Guizhou Province, China. Journal of East China Normal 
University (Natural Science), 22(5), 43-52. (in Chinese with English abstract) [罗祖奎, 刘伦沛, 王云, 牛俊英, 刘立 波, 李丽 (2013) 贵州省云台山鸟类群落特征. 华东师范 大学学报(自然科学版), 22(5), 43-52.]

Ma M, Xu F, Chundawat RS, Jumabay K, Wu YQ, Aizezi, Zhu MH (2006) Camera trapping of snow leopards for the photo capture rate and population size in the Muzat Valley of Tianshan Mountains. Acta Zoologica Sinica, 52, 788-793. (in Chinese with English abstract) [马鸣, 徐峰, Chundawat RS, Kubanych Jumabay, 吴逸群, 艾则孜, 朱玛洪 (2006) 利用自动照相术获得天山雪豹拍摄率与个体数量. 动物 学报, 52, 788-793.]

Ma SL, Harris RB (1996) Use of remote camera systems to document wildlife species presence in forested areas of Yunnan. Zoological Research, 17, 360-370. (in Chinese) [马世来, Richard B. Harris (1996) 自动感应照像系统在野 生动物调查中的应用. 动物学研究, 17, 360-370.]

Meek PD, Ballard G, Claridge A, Kays R, Moseby K, O’Brien T, O’Connell A, Sanderson J, Swann DE, Tobler M, Townsend S (2014) Recommended guiding principles for reporting on camera trapping research. Biodiversity Conservation, 23, 2321-2343.

O’Brien TG, Kinnaird MF (2008) A picture is worth a thousand words: The application of camera trapping to the study of birds. Bird Conservation International, 18, S144-S162.

O’Connell AF, Nichols JD, Karanth KU (2011) Camera Traps in Animal Ecology: Methods and Analyses. Springer, New York.

Pang XF (2003) Studies on Biodiversity of the Guangdong Nanling National Nature Reserve. Guangdong Science and Technology Press, Guangzhou. (in Chinese) [ 庞雄飞 (2003) 广东南岭国家级自然保护区生物多样性研究. 广 东科技出版社, 广州.]

Pearson OP (1959) A traffic survey of Microtus-Reithrodontomys runways. Journal of Mammalogy, 40, 169-180.

Pei K (1995) Activity rhythm of the spinous country rat (Niviventer coxingi) in Taiwan. Zoological Studies, 34, 55-58.

Piratelli A (2003) Mesh size and bird capture rates in Mato Grosso do Sul State, Brazil. Brazilian Journal of Biology, 63, 105-111.

Rappole JH, Winker K, Powell GVN (1998) Migratory birds habitat use in southern Mexico: Mist nets versus point counts. Journal of Field Ornithology, 69, 635-643.

Rowcliffe JM, Kays R, Kranstauber B, Carbone C, Jansen PA (2014) Quantifying levels of animal activity using camera trap data. Methods in Ecology and Evolution, 5, 1170-1179.

Si XF, Ding P (2014) Camera trap survey on population dynamics of mammals and birds in Gutianshan Forest Dynamics Plot, eastern China. Biodiversity Science, 22, 819-822. (in Chinese) [斯幸峰, 丁平 (2014) 古田山森林动态监测 样地内鸟兽种群动态的红外相机监测. 生物多样性, 22, 819-822.]

Steenweg R, Hebblewhite M, Kays R, Ahumada J, Fisher JT,
Burton C, Townsends SE, Carbone C, Rowcliffe JM, Whittington J, Brodie J, Royle JA, Switalski A, Clevenger AP, Heim N, Rich LN (2017) Scaling-up camera traps: Monitoring the planet's biodiversity with networks of remote sensors. Frontier in Ecology and Environment, 15, 26-34.

Tian HZ, Xing FW (2008) Elevational diversity patterns of orchids in Nanling National Nature Reserve, northern Guangdong Province. Biodiversity Science, 16, 75-82. (in Chinese with English abstract) [田怀珍, 邢福武 (2008) 南 岭国家级自然保护区兰科植物物种多样性的海拔梯度格 局. 生物多样性, 16, 75-82.]

Tobler MW, Carrillo-Percastegui SE, Pitman RL, Mares R, Powell G (2008) Further notes on the analysis of mammal inventory data collected with camera traps. Animal Conservation, 11, 187-189.

Verner J, Ritter LV (1985) A comparison of transects and point counts in oak-pine woodlands of California. Condor, 87, 47-68.

Wallihan AG, Wallihan MAH (1906) Camera Shots at Big Game. Doubleday, Page \& Co, New York.

Wang FG, Chen ZM, Chen HF, Xing FW, Dong AQ (2013) Flora and Vegetation of Nanling National Nature Reserve. Huazhong University of Science \& Technology Press, Wuhan. (in Chinese) [王发国, 陈振明, 陈红锋, 邢福武, 董安强 (2013) 南岭国家级自然保护区植物区系与植被. 华中科技大学出版社, 武汉.]

Wang GH, Shi ZP, Liu XJ, Zhou QH, Xiao ZS (2014) Camera trap survey of mammals and birds in Huaping National Nature Reserve, Guangxi. Biodiversity Science, 22, 758-787. (in Chinese) [汪国海, 施泽攀, 刘秀菊, 周岐海, 肖治术 (2014) 花坪保护区鸟兽物种的红外相机初步监测. 生物 多样性, 22, 785-787.]

Wang Y, Finch DM (2002) Consistency of mist netting and point counts in assessing landbird species richness and relative abundance during migration. Condor, 104, 59-72.

Whitman AA, Hagan JM III, Brokaw NVL (1997) A compareison of two bird survey techniques used in a subtropical forest. Condor, 99, 955-965.

Wu F, Yang XJ (2008) Application of point count method in forest birds survey. Chinese Journal of Ecology, 27, 2240-2244. (in Chinese with English abstract) [吴飞, 杨晓 君 (2008) 样点法在森林鸟类调查中的运用. 生态学杂 志, 27, 2240-2244.]

Xiao ZS, Wu LF, Tang LF, Lu XL, Huang ZL, Ye WH, Huang XQ (2014a) Camera trap survey of mammals and birds in Dinghushan forest dynamics plot, southern China. Biodiversity Science, 22, 823-825. (in Chinese) [肖治术, 吴林芳, 唐林芳, 卢学理, 黄忠良, 叶万辉, 黄小群 (2014a) 运用红外相机对鼎湖山森林动态监测样地鸟兽 的初步调查. 生物多样性, 22, 823-825.]

Xiao ZS, Li XH, Wang XZ, Zhou QH, Quan RC, Shen XL, Li $S$ (2014b) Developing camera-trapping protocols for 
wildlife monitoring in Chinese forests. Biodiversity Science, 22, 704-711. (in Chinese with English abstract) [肖治术, 李欣海, 王学志, 周岐海, 权锐昌, 申小莉, 李晟 (2014b) 探讨我国森林野生动物红外相机监测规范. 生 物多样性, 22, 704-711.]

Xu L, Zhang ZW, Ding CQ (2003) Line transect methods in avian census. Chinese Journal of Ecology, 22, 127-130. (in Chinese with English abstract) [许龙, 张正旺, 丁长青 (2003) 样线法在鸟类数量调查中的运用. 生态学杂志, 22, 127-130.]

Zhang Q (2011) Avian Communities and Mixed-species Bird Flocks Associated with Successional Stands in Subtropical Forest in Dinghushan. PhD dissertation, South China Botanical Garden, Chinese Academy of Sciences, Guangzhou. (in Chinese with English abstract) [张强 (2011) 鼎湖山森林 演替不同阶段鸟类群落及混合群研究. 博士学位论文, 中国科学院华南植物园, 广州.]

Zhao ZJ (2001a) A Handbook of the Birds of China (Volume I): Non-passerines. Science and Technique Press of Jilin Province, Changchun. (in Chinese with English abstract) [ 赵 正阶 (2001a) 中国鸟类志 (上卷): 非雀形目. 吉林科学 技术出版社, 长春.]

Zhao ZJ (2001b) A Handbook of the Birds of China (Volume II): Passerines. Science and Technique Press of Jilin Province, Changchun. (in Chinese with English abstract) [ 赵 正阶(2001b) 中国鸟类志 (下卷): 雀形目. 吉林科学技术 出版社, 长春.]

Zheng GM (2012) Ornithology, 2nd edn. Beijing Normal University Press, Beijing. (in Chinese) [郑光美 (2012) 鸟 类学(第二版). 北京师范大学出版社, 北京.]

Zhou F (1986) On the breeding bird communities of Dinghushan. Tropical Subtropical Forest Ecosystem, 4, 79-91. (in Chinese with English abstract) [周放 (1986) 鼎 湖山森林繁殖鸟类群落的研究. 热带亚热带森林生态系 统研究, 4, 79-91.]

Zhu SY, Duan F, Li S (2017) Promoting diversity inventory and monitoring of birds through the camera-trapping network in China: Status, challenges and future outlook. Biodiversity Science, 25, 1114-1122. (in Chinese with English abstract) [朱淑怡, 段菲, 李晟 (2017) 基于红外 相机网络促进我国鸟类多样性监测: 现状, 问题与前景. 生物多样性, 25, 1114-1122.]

Zou FS (2002) A Study on Bird Communities of Tropical Forest at Jianfengling on Hainan Island. PhD dissertation, Sun Yat-sen University, Guangzhou. (in Chinese with English abstract) [邹发生 (2002) 海南岛尖峰岭热带森林 鸟类群落研究. 博士学位论文, 中山大学, 广州.]

Zou FS, Chen GZ (2003) Application of mist net in studies of forest bird communities. Chinese Journal of Applied Ecology, 14, 1557-1560. (in Chinese with English abstract) [邹 发生, 陈桂珠 (2003) 雾网在森林鸟类群落研究中的应 用. 应用生态学报, 14, 1557-1560.]

Zou FS, Chen GZ (2004) A study of understory bird communities in tropical mountain rain forest of Jianfengling, Hainan Island, China. Acta Ecologica Sinica, 24, 510-516. (in Chinese with English abstract) [邹发生, 陈桂珠 (2004) 海南岛尖峰岭热带山地雨林林下鸟类群落研究. 生态学 报, 24, 510-516.]

Zou FS, Chen GZ, Yang QF (2010) Impacts of bird abundance, activity height and light intensity on the number of birds captured by mist netting. Chinese Birds, 1, 221-229.

Zou FS, Chen GZ, Yang QF, Li YD (2012) Bird species richness along an elevational gradient in forest at Jianfengling, Hainan Island, China. Zoological Studies, 51, 362-371.

Zou FS, Yang QF (2005) A study of understory bird communities in an artificial forest in Heshan, Guangdong, China. Acta Ecologica Sinica, 25, 3323-3328. (in Chinese with English abstract) [邹发生, 杨琼芳 (2005) 广东鹤山丘陵 人工林林下鸟群落研究. 生态学报, 25, 3323-3328.]

(责任编委: 丁 平 责任编辑: 间文杰)

\section{附录 Supplementary Material}

附录1 广东南岭和车八岭国家级自然保护区网捕和红外相机监测鸟类数量以及身体大小

Appendix 1 Bird number by camera trapping or mist net and bird body size at Nanling National Nature Reserve and Chebaling National Nature Reserve, Guangdong http://www.biodiversity-science.net/fileup/PDF/2017275-1.pdf 
张倩雯, 龚粤宁, 宋相金, 王新财, 杨昌滕, 束祖飞, 邹发生. 红外相机技术与其他几种森林鸟类多样性调查方法的比较. 生物多样性, 2018,26 (3): $229-237$. http://www.biodiversity-science.net/CN/10.17520/biods.2017275

附录1 广东南岭和车八岭国家级自然保护区网捕和红外相机监测鸟类数量以及身体大小

Appendix 1 Bird number by camera trapping or mist net and bird body size at Nanling National Nature Reserve and Chebaling National Nature Reserve, Guangdong

\begin{tabular}{|c|c|c|c|c|c|c|c|c|c|}
\hline \multirow[b]{2}{*}{$\begin{array}{l}\text { 物种名 } \\
\text { Species list }\end{array}$} & \multicolumn{2}{|c|}{ 南岭 Nanling } & \multicolumn{2}{|c|}{ 车八岭 Chebaling } & \multirow[b]{2}{*}{$\begin{array}{l}\text { 体重 } \\
\text { Weight }\end{array}$} & \multirow[b]{2}{*}{$\begin{array}{l}\text { 体长 } \\
\text { Body length }\end{array}$} & \multirow[b]{2}{*}{$\begin{array}{l}\text { 翅长 } \\
\text { Wing length }\end{array}$} & \multirow[b]{2}{*}{$\begin{array}{l}\text { 跗蹠 } \\
\text { Tarsus length }\end{array}$} & \multirow[b]{2}{*}{$\begin{array}{l}\text { 参考文献 } \\
\text { References }\end{array}$} \\
\hline & $\begin{array}{r}\text { 网捕 } \\
\text { Mist net }\end{array}$ & $\begin{array}{c}\text { 红外相机 } \\
\text { Camera } \\
\text { trapping }\end{array}$ & $\begin{array}{c}\text { 网捕 } \\
\text { Mist net }\end{array}$ & $\begin{array}{c}\text { 红外相机 } \\
\text { Camera } \\
\text { trapping }\end{array}$ & & & & & \\
\hline 松雀鹰 Accipiter virgatus & 1 & 2 & & & $182.5 \pm 7.54(4)$ & $324.33 \pm 26.96(3)$ & $190.75 \pm 12.15(4)$ & $53.25 \pm 4.19(4)$ & 赵正阶, 2001a \\
\hline 日本松雀鹰 A. gularis & & & 1 & 0 & $119.5 \pm 20.28(4)$ & $256.75 \pm 15.55(6)$ & $146.6 \pm 26.13(6)$ & $55.5 \pm 1.52(6)$ & 本研究 \\
\hline 白眉山府鸪 Arborophila gingica & 0 & 324 & 0 & 42 & 253(1) & $300(1)$ & $148 \pm 2.52(3)$ & $42.67 \pm 1.2(3)$ & $\begin{array}{c}\text { 赵正阶, 2001a } \\
\dagger\end{array}$ \\
\hline 白㸡 Lophura nycthemera & 0 & 1,242 & 0 & 1,069 & $\begin{array}{l}1,448.75 \pm \\
203.94(4)\end{array}$ & $871 \pm 115.09(4)$ & $261.75 \pm 11.95(4)$ & $91.5 \pm 3.23(4)$ & 赵正阶, 2001a \\
\hline 黄腹角雉 Tragopan caboti & 0 & 181 & & & $1,400(1)$ & $653.5 \pm 46.5(2)$ & $215.75 \pm 8.92(4)$ & $64.25 \pm 2.66(4)$ & 赵正阶, 2001a \\
\hline 环颈雉 Phasianus colchicus & & & 0 & 2 & $1,196 \pm 171.52(4)$ & $700 \pm 63.88(4)$ & $222 \pm 7.95(4)$ & $64.38 \pm 5.11(4)$ & 赵正阶, 2001a \\
\hline 灰胸竹鸡 Bambusicola thoracicus & 0 & 32 & 0 & 90 & $277.25 \pm 33.77(4)$ & $281.29 \pm 4.32(16)$ & $133.05 \pm 1.47(17)$ & $46.59 \pm 0.94(17)$ & 本研究 \\
\hline 丘㦷 Scolopax rusticola & 0 & 28 & 0 & 11 & $271.5 \pm 30.42(4)$ & $362.5 \pm 20.87(4)$ & $195.25 \pm 7.41(4)$ & $38.5 \pm 2.18(4)$ & 赵正阶, 2001a \\
\hline 绿翅金鸭 Chalcophaps indica & 0 & 1 & & & $94.35 \pm 9.22(5)$ & $233.03 \pm 7.39(12)$ & $141.67 \pm 2.23(12)$ & $26.96 \pm 1.37(12)$ & 本研究 \\
\hline 红翅风头鹃 Clamator coromandus & & & 1 & 0 & $60.79(1)$ & $323(1)$ & $156(1)$ & $28 \pm 0(1)$ & 本研究 \\
\hline 褐翅鸦鹃 Centropus sinensis & & & 0 & 1 & $300.5 \pm 31.31(4)$ & $473.75 \pm 25.77(4)$ & $215 \pm 6.95(4)$ & $56 \pm 1.47(4)$ & 赵正阶, 2001a \\
\hline 小鸦鹃 C. bengalensis & & & 0 & 1 & $124.25 \pm 18.23(4)$ & $352.75 \pm 22.17(4)$ & $157.25 \pm 11.95(4)$ & $42 \pm 2.2(4)$ & 赵正阶, 2001a \\
\hline 中杜鹃 Cuculus saturatus & 1 & 0 & & & $104.25 \pm 14.26(4)$ & $297 \pm 25.37(4)$ & $200 \pm 8.51(4)$ & $21 \pm 1.29(4)$ & 赵正阶, 2001a \\
\hline 红 (东方) 角鸮 Otus sunia & 1 & 0 & & & $73 \pm 13.4(4)$ & $182.75 \pm 7.31(4)$ & $145.5 \pm 2.63(4)$ & $25.75 \pm 1.03(4)$ & 赵正阶, 2001a \\
\hline 领角鸮 O. lettia & 0 & 1 & 1 & 0 & 136.67(1) & $231.65 \pm 3.47(4)$ & $165.98 \pm 4.75(4)$ & $41.06 \pm 3.71(4)$ & 本研究 \\
\hline 斑头鸺留 Glaucidium cuculoides & 1 & 1 & 3 & 0 & $193.25 \pm 26.18(4)$ & $252.75 \pm 4.57(4)$ & $162 \pm 8.98(4)$ & $29.75 \pm 1.49(4)$ & 赵正阶, 2001a \\
\hline 领㭤留 G. brodiei & 11 & 5 & 1 & 0 & $38.15(1)$ & $160.5 \pm 2.5(2)$ & $87 \pm 3(2)$ & $22 \pm 3(2)$ & 本研究 \\
\hline 普通夜鹰 Caprimulgus indicus & 0 & 16 & & & $94.75 \pm 7.53(4)$ & $268 \pm 4.81(4)$ & $206 \pm 4.78(4)$ & $16.25 \pm 1.31(4)$ & 赵正阶, 2001a \\
\hline
\end{tabular}


张倩雯，龚粤宁，宋相金，王新财，杨昌腾，束祖飞，邹发生. 红外相机技术与其他几种森林鸟类多样性调查方法的比较. 生物多样性, 2018, 26 (3): 229-237. http://www.biodiversity-science.net/CN/10.17520/biods.2017275

\begin{tabular}{|c|c|c|c|c|c|c|c|c|c|}
\hline \multirow[b]{2}{*}{$\begin{array}{l}\text { 物种名 } \\
\text { Species list }\end{array}$} & \multicolumn{2}{|c|}{ 南岭 Nanling } & \multicolumn{2}{|c|}{ 车八岭 Chebaling } & \multirow[b]{2}{*}{$\begin{array}{l}\text { 体重 } \\
\text { Weight }\end{array}$} & \multirow[b]{2}{*}{$\begin{array}{l}\text { 体长 } \\
\text { Body length }\end{array}$} & \multirow[b]{2}{*}{$\begin{array}{l}\text { 翅长 } \\
\text { Wing length }\end{array}$} & \multirow[b]{2}{*}{$\begin{array}{l}\text { 跗蹠 } \\
\text { Tarsus length }\end{array}$} & \multirow[b]{2}{*}{$\begin{array}{l}\text { 参考文献 } \\
\text { References }\end{array}$} \\
\hline & $\begin{array}{c}\text { 网捕 } \\
\text { Mist net }\end{array}$ & $\begin{array}{c}\text { 红外相机 } \\
\text { Camera } \\
\text { trapping }\end{array}$ & $\begin{array}{c}\text { 网捕 } \\
\text { Mist net }\end{array}$ & $\begin{array}{c}\text { 红外相机 } \\
\text { Camera } \\
\text { trapping }\end{array}$ & & & & & \\
\hline 红头咬鹃 Harpactes erythrocephalus & 5 & 1 & 13 & 0 & 108.5(1) & $328.95 \pm 3.68(4)$ & $151.75 \pm 3.39(4)$ & $20.78 \pm 0.45(4)$ & 本研究 \\
\hline 大拟豚木鸟 Megalaima virens & 0 & 1 & & & $187.5 \pm 17.11(4)$ & $315.75 \pm 8.5(4)$ & $143 \pm 2.97(4)$ & $32.75 \pm 1.11(4)$ & 赵正阶, 2001a \\
\hline 黑眉拟豚木鸟 M. oorti & 2 & 0 & & & $91.5 \pm 7(2)$ & $253.06 \pm 2.77(15)$ & $111.42 \pm 0.77(15)$ & $32.91 \pm 0.57(15)$ & 本研究 \\
\hline 黄嘴栗啄木鸟 Blythipicus pyrrhotis & 3 & 8 & 6 & 1 & $103.8 \pm 21.15(3)$ & $280.6 \pm 6.19(5)$ & $150.88 \pm 2.82(5)$ & $32.67 \pm 1.23(5)$ & 本研究 \\
\hline 栗啄木鸟 Celeus brachyurus & 1 & 0 & 3 & 0 & $64.04 \pm 14.24(2)$ & $242.67 \pm 22.75(3)$ & $121.33 \pm 5.55(3)$ & $22 \pm 3.06(3)$ & 本研究 \\
\hline 灰头绿豚木鸟 Picus canus & 0 & 5 & 3 & 6 & 136.23(1) & $301.33 \pm 12.98(3)$ & $148 \pm 1(3)$ & $48.06 \pm 16.97(3)$ & 本研究 \\
\hline 斑姬豚木鸟 Picumnus innominatus & & & 1 & 0 & $10.52 \pm 0.45(12)$ & $96.97 \pm 1.38(18)$ & $57.15 \pm 0.76(18)$ & $13.17 \pm 0.68(14)$ & 本研究 \\
\hline 仙八色冻 Pitta nympha & & & 0 & 1 & $54.25 \pm 5.27(4)$ & $191.25 \pm 7.65(4)$ & $117.25 \pm 3.68(4)$ & $39 \pm 1.08(4)$ & 赵正阶, 2001b \\
\hline 赤红山椒鸟 Pericrocotus flammeus & & & 1 & 0 & 20.86(1) & $212.75 \pm 1.75(2)$ & $97.65 \pm 0.35(2)$ & $20.87 \pm 1.03(2)$ & 本研究 \\
\hline 灰喉山椒鸟 P. solaris & & & 23 & 0 & $15.75 \pm 0.65(2)$ & $150.87 \pm 22.9(7)$ & $78.31 \pm 4.67(7)$ & $17.33 \pm 1.12(7)$ & 本研究 \\
\hline 白头鹎 Pycnonotus sinensis & & & 0 & 1 & $28.52 \pm 0.82(35)$ & $178.3 \pm 1.86(103)$ & $84.51 \pm 0.47(107)$ & $22.28 \pm 0.26(106)$ & 本研究 \\
\hline 黑短脚䴗 Hypsipetes leucocephalus & & & 1 & 0 & $46.26 \pm 1.41(6)$ & $240.9 \pm 2.02(25)$ & $113.68 \pm 1.01(25)$ & $20.88 \pm 0.26(25)$ & 本研究 \\
\hline 绿翅短脚軼 H. mcclellandii & 10 & 4 & 22 & 0 & $36.23 \pm 1.32(17)$ & $221.87 \pm 1.98(35)$ & $102.54 \pm 1.37(35)$ & $20.21 \pm 0.4(35)$ & 本研究 \\
\hline 栗背短脚軠 Hemixos castanonotus & 13 & 4 & 36 & 1 & $72.24 \pm 13.44(30)$ & $200.6 \pm 3.57(96)$ & $99.41 \pm 0.68(96)$ & $20.09 \pm 0.73(88)$ & 本研究 \\
\hline 橙腹叶軼 Chloropsis hardwickii & & & 3 & 0 & $27.97 \pm 1.23(2)$ & $192 \pm 3(2)$ & $87.5 \pm 3.5(2)$ & $20.5 \pm 0.5(2)$ & 本研究 \\
\hline 钩嘴林鵙 Tephrodornis gularis & & & 5 & 0 & $36.82 \pm 1.03(13)$ & $212.35 \pm 1.86(29)$ & $118.13 \pm 0.81(29)$ & $24.25 \pm 0.22(28)$ & 本研究 \\
\hline 灰树鹊 Dendrocitta formosae & 4 & 8 & 73 & 79 & $88.5 \pm 13.5(2)$ & $327.93 \pm 7.95(17)$ & $136.08 \pm 2.75(17)$ & $30.04 \pm 1.15(16)$ & 本研究 \\
\hline
\end{tabular}


张倩雯, 龚粤宁, 宋相金, 王新财, 杨昌腾, 束祖飞, 䢹发生. 红外相机技术与其他几种森林鸟类多样性调查方法的比较. 生物多样性, 2018,26 (3): $229-237$. http://www.biodiversity-science.net/CN/10.17520/biods.2017275

\begin{tabular}{|c|c|c|c|c|c|c|c|c|c|}
\hline \multirow[b]{2}{*}{ 物种名 } & \multicolumn{2}{|c|}{ 南岭 Nanling } & \multicolumn{2}{|c|}{ 车八岭 Chebaling } & \multirow[b]{2}{*}{$\begin{array}{l}\text { 体重 } \\
\text { Weight }\end{array}$} & \multirow[b]{2}{*}{$\begin{array}{l}\text { 体长 } \\
\text { Body length }\end{array}$} & \multirow[b]{2}{*}{$\begin{array}{l}\text { 翅长 } \\
\text { Wing length }\end{array}$} & \multirow[b]{2}{*}{$\begin{array}{l}\text { 跗蹠 } \\
\text { Tarsus length }\end{array}$} & \multirow[b]{2}{*}{$\begin{array}{l}\text { 参考文献 } \\
\text { References }\end{array}$} \\
\hline & $\begin{array}{c}\text { 网捕 } \\
\text { Mist net }\end{array}$ & $\begin{array}{c}\text { 红外相机 } \\
\text { Camera } \\
\text { trapping }\end{array}$ & $\begin{array}{c}\text { 网捕 } \\
\text { Mist net }\end{array}$ & $\begin{array}{c}\text { 红外相机 } \\
\text { Camera } \\
\text { trapping }\end{array}$ & & & & & \\
\hline 红嘴蓝鹊 Urocissa erythrorhyncha & 0 & 16 & 0 & 4 & $176.75 \pm 16.45(4)$ & $580.75 \pm 37.16(4)$ & $191.75 \pm 8.33(4)$ & $44 \pm 2.35(4)$ & 赵正阶, 2001b \\
\hline 红胁蓝尾鸲 Tarsiger cyanurus & 16 & 83 & 25 & 22 & $12.49 \pm 0.17(77)$ & $133.47 \pm 0.61(284)$ & $80.32 \pm 2.53(284)$ & $23.44 \pm 0.11(281)$ & 本研究 \\
\hline 白尾地鸲 Cinclidium leucurum & 4 & 2 & & & $24.75 \pm 1.03(4)$ & $163.25 \pm 6.47(4)$ & $90 \pm 3.85(4)$ & $26.5 \pm 1.19(4)$ & 赵正阶, 2001b \\
\hline 红尾歌鸲 Luscinia sibilans & & & 2 & 0 & $14.4 \pm 2.09(4)$ & $131.64 \pm 3.35(54)$ & $71.21 \pm 0.91(53)$ & $27.24 \pm 0.39(43)$ & 本研究 \\
\hline 日本歌鸲 Erithacus akahige & & & 1 & 0 & 17(1) & $136.5 \pm 3.5(2)$ & $74.75 \pm 1.49(4)$ & $28.5 \pm 0.96(4)$ & $\begin{array}{l}\text { 赵正阶, 2001b; } \\
\text { Dunning, } 2007\end{array}$ \\
\hline 白额燕尾 Enicurus leschenaulti & 0 & 6 & 0 & 3 & $43.8 \pm 1.76(5)$ & $256.31 \pm 11.47(9)$ & $106.87 \pm 2.61(9)$ & $34.27 \pm 1.38(9)$ & 本研究 \\
\hline 斑背燕尾 E. maculatus & 12 & 10 & & & $40.92 \pm 2.61(3)$ & $236.06 \pm 8.13(8)$ & $103.76 \pm 2.09(9)$ & $33.56 \pm 0.4(9)$ & 本研究 \\
\hline 灰背燕尾 E. schistaceus & 2 & 2 & & & $25.7 \pm 1.58(5)$ & $217.67 \pm 7.07(8)$ & $98.08 \pm 2(8)$ & $31.7 \pm 0.4(8)$ & 本研究 \\
\hline 小燕尾 E. scouleri & 0 & 1 & & & $17.25 \pm 1.6(4)$ & $124.25 \pm 5.86(4)$ & $74.25 \pm 3.59(4)$ & $23.5 \pm 0.96(4)$ & 赵正阶, 2001b \\
\hline 紫啸鸫 Myophonus caeruleus & 7 & 737 & 1 & 7 & $147.75 \pm 43.61(5)$ & $263.67 \pm 12.43(16)$ & $158.42 \pm 2.9(16)$ & $52.43 \pm 2.33(16)$ & 本研究 \\
\hline 橙头地鸫 Zoothera citrina & 0 & 16 & & & $49.57 \pm 2.5(17)$ & $186.45 \pm 9.1(20)$ & $108.41 \pm 5.2(20)$ & $35.18 \pm 0.9(20)$ & 本研究 \\
\hline 虎斑地冻 Z. dauma & 0 & 71 & 2 & 62 & $105.22(1)$ & $305.02 \pm 1.98(14)$ & $166.83 \pm 1.18(14)$ & $40.15 \pm 0.29(14)$ & 本研究 \\
\hline 白眉地冻 Z. sibirica & 0 & 11 & 0 & 4 & $69.5 \pm 7.47(4)$ & 213.86(1) & 121(1) & $33.68 \pm 0(1)$ & $\begin{array}{l}\text { 本研究; 赵正 } \\
\text { 阶, 2001b }\end{array}$ \\
\hline 灰背鸫 Turdus hortulorum & 0 & 4 & 61 & 2 & $60.52 \pm 1.63(10)$ & $205.61 \pm 3.23(32)$ & $113.76 \pm 1.51(33)$ & $32.07 \pm 1.05(33)$ & 本研究 \\
\hline 白眉鸫 T. obscurus & 0 & 5 & 0 & 2 & $78.57(1)$ & $215.17 \pm 10.29(3)$ & $120.53 \pm 4.61(3)$ & $33.19 \pm 0.92(3)$ & 本研究 \\
\hline 白腹蓝姬䳜 Cyanoptila cyanomelana & & & 1 & 0 & $23.25 \pm 2.32(4)$ & $154.5 \pm 6.98(4)$ & $91.5 \pm 2.53(4)$ & $17.25 \pm 1.31(4)$ & 赵正阶, 2001b \\
\hline 白喉林㰸 Rhinomyias brunneatus & 10 & 0 & & & $16.54 \pm 0.74(7)$ & $138.08 \pm 3.31(13)$ & $70.38 \pm 2.68(13)$ & $18.9 \pm 0.59(13)$ & 本研究 \\
\hline 灰纹䳜 Muscicapa griseisticta & & & 1 & 0 & $17.33 \pm 2.91(3)$ & $130 \pm 12(2)$ & $83.25 \pm 2.95(4)$ & $13.5 \pm 0.76(3)$ & 赵正阶, 2001b \\
\hline
\end{tabular}


张倩雯, 龚粤宁, 宋相金, 王新财, 杨昌腾, 束祖飞, 䢹发生. 红外相机技术与其他几种森林鸟类多样性调查方法的比较. 生物多样性, 2018,26 (3): $229-237$. http://www.biodiversity-science.net/CN/10.17520/biods.2017275

\begin{tabular}{|c|c|c|c|c|c|c|c|c|c|}
\hline \multirow[b]{2}{*}{$\begin{array}{l}\text { 物种名 } \\
\text { Species list }\end{array}$} & \multicolumn{2}{|c|}{ 南岭 Nanling } & \multicolumn{2}{|c|}{ 车八岭 Chebaling } & \multirow[b]{2}{*}{$\begin{array}{l}\text { 体重 } \\
\text { Weight }\end{array}$} & \multirow[b]{2}{*}{$\begin{array}{l}\text { 体长 } \\
\text { Body length }\end{array}$} & \multirow[b]{2}{*}{$\begin{array}{l}\text { 翅长 } \\
\text { Wing length }\end{array}$} & \multirow[b]{2}{*}{$\begin{array}{l}\text { 跗蹠 } \\
\text { Tarsus length }\end{array}$} & \multirow[b]{2}{*}{$\begin{array}{l}\text { 参考文献 } \\
\text { References }\end{array}$} \\
\hline & $\begin{array}{l}\text { 网捕 } \\
\text { Mist net }\end{array}$ & $\begin{array}{c}\text { 红外相机 } \\
\text { Camera } \\
\text { trapping }\end{array}$ & $\begin{array}{l}\text { 网捕 } \\
\text { Mist net }\end{array}$ & $\begin{array}{c}\text { 红外相机 } \\
\text { Camera } \\
\text { trapping }\end{array}$ & & & & & \\
\hline 鸲姬釷 Ficedula mugimaki & 1 & 0 & 3 & 0 & $11.2(1)$ & $124.41 \pm 0.85(56)$ & $73.12 \pm 0.43(56)$ & $17.26 \pm 0.1(48)$ & 本研究 \\
\hline 小仙骵 Niltava macgrigoriae & 20 & 0 & & & $13.53 \pm 0.86(18)$ & $126.65 \pm 4.1(24)$ & $68.42 \pm 1.71(25)$ & $20.15 \pm 0.42(24)$ & 本研究 \\
\hline 棕腹大仙䳜 N. davidi & 1 & 0 & & & $20.04 \pm 1.03(13)$ & $158.49 \pm 3.55(17)$ & $86.68 \pm 1.59(17)$ & $22.98 \pm 0.39(16)$ & 本研究 \\
\hline 海南蓝仙䳜 Cyornis hainanus & 4 & 0 & & & $11.88 \pm 0.16(60)$ & $130.83 \pm 1.03(66)$ & $68.2 \pm 0.48(66)$ & $18.94 \pm 0.83(66)$ & 本研究 \\
\hline 铜蓝銤 Eumyias thalassinus & 0 & 1 & & & $15.46(1)$ & $162.2 \pm 6.2(2)$ & $86.1 \pm 2.1(2)$ & $17.12 \pm 1.12(2)$ & 本研究 \\
\hline 小黑领噪鹒 Garrulax monileger & 1 & 0 & 7 & 0 & $67.56 \pm 3.25(21)$ & $263.1 \pm 4.51(37)$ & $117.87 \pm 1.52(37)$ & $40.03 \pm 0.79(37)$ & 本研究 \\
\hline 黑领噪鹛 G. pectoralis & 1 & 259 & 12 & 704 & $119.18 \pm 6.81(12)$ & $303.58 \pm 4.83(48)$ & $128.56 \pm 2.36(48)$ & $48.59 \pm 0.51(47)$ & 本研究 \\
\hline 黑喉噪䴙 G. chinensis & 0 & 5 & 0 & 1 & $88.25 \pm 4.44(4)$ & $257.25 \pm 15.8(4)$ & $110.25 \pm 3.17(4)$ & $40.5 \pm 1.76(4)$ & 赵正阶, 2001b \\
\hline 褐胸噪鹋 G. maesi & 3 & 156 & & & $77.67 \pm 6.22(3)$ & $267.64 \pm 7.46(11)$ & $123 \pm 1.43(11)$ & $47.05 \pm 1.21(11)$ & 本研究 \\
\hline 棕噪鸽 G. poecilorhynchus & 3 & 7 & & & $73.4 \pm 5.29(2)$ & $298.42 \pm 6.16(12)$ & $116.67 \pm 1.25(12)$ & $44.58 \pm 0.95(12)$ & 本研究 \\
\hline 画眉 G. canorus & 3 & 4 & & & $55.82 \pm 1.76(14)$ & $231.85 \pm 4.8(34)$ & $91.34 \pm 0.62(37)$ & $40.2 \pm 0.41(37)$ & 本研究 \\
\hline 赤尾噪鹤 G. milnei & 1 & 8 & & & $66.61(1)$ & $267(1)$ & $108(1)$ & $40.5 \pm 0(1)$ & 本研究 \\
\hline 白颉噪鹃 G. sannio & 0 & 2 & & & $62.11 \pm 2.26(5)$ & $213.25 \pm 9.09(8)$ & $93.88 \pm 2.29(8)$ & $38.03 \pm 0.97(8)$ & 本研究 \\
\hline 棕颈钩嘴鹋 Pomatorhinus ruficollis & 15 & 24 & 3 & 3 & $25.73 \pm 0.33(88)$ & $172.4 \pm 1.28(123)$ & $74.22 \pm 0.71(122)$ & $30.99 \pm 0.28(123)$ & 本研究 \\
\hline 斑胸钩嘴鹋 P. erythrocnemis & 1 & 56 & 4 & 17 & $61.02 \pm 1.77(6)$ & $223.89 \pm 8.95(9)$ & $88.83 \pm 1.25(9)$ & $38.63 \pm 0.84(11)$ & 本研究 \\
\hline 红头穗鸲 Stachyris ruficeps & 7 & 0 & 2 & 0 & $8.81 \pm 0.11(81)$ & $107.4 \pm 0.78(116)$ & $51.24 \pm 0.36(116)$ & $20.1 \pm 0.16(114)$ & 本研究 \\
\hline 红嘴相思鸟 Leiothrix lutea & 57 & 30 & 42 & 2 & $19.58 \pm 0.23(92)$ & $141.9 \pm 0.79(135)$ & $67.34 \pm 0.26(136)$ & $26.57 \pm 0.16(136)$ & 本研究 \\
\hline 金胸雀倜 Alcippe chrysotis & 1 & 0 & & & $6.84 \pm 0.17(10)$ & $98.8 \pm 3.61(10)$ & $50.8 \pm 0.59(10)$ & $22.27 \pm 0.45(11)$ & 本研究 \\
\hline 褐顶雀倜 A. brunnea & 20 & 2 & & & $17.23 \pm 0.39(14)$ & $125.45 \pm 5.04(19)$ & $58.79 \pm 0.89(19)$ & $24.95 \pm 0.51(18)$ & 本研究 \\
\hline 灰眶雀鹘 A. morrisonia & 149 & 29 & 231 & 26 & $16.5 \pm 1.13(275)$ & $128.1 \pm 0.92(354)$ & $64.25 \pm 1.77(357)$ & $23.06 \pm 0.58(358)$ & 本研究 \\
\hline 栗耳风鸤 Yuhina castaniceps & 4 & 1 & 24 & 0 & $12.49 \pm 0.33(9)$ & $137.29 \pm 1.53(32)$ & $65.1 \pm 0.4(32)$ & $19.2 \pm 0.22(32)$ & 本研究 \\
\hline
\end{tabular}


张倩雯, 龚粤宁, 宋相金, 王新财, 杨昌腾, 束祖飞, 䢹发生. 红外相机技术与其他几种森林鸟类多样性调查方法的比较. 生物多样性, 2018,26 (3): $229-237$. http://www.biodiversity-science.net/CN/10.17520/biods.2017275

\begin{tabular}{|c|c|c|c|c|c|c|c|c|c|}
\hline \multirow[b]{2}{*}{$\begin{array}{l}\text { 物种名 } \\
\text { Species list }\end{array}$} & \multicolumn{2}{|c|}{ 南岭 Nanling } & \multicolumn{2}{|c|}{ 车八岭 Chebaling } & \multirow[b]{2}{*}{$\begin{array}{l}\text { 体重 } \\
\text { Weight }\end{array}$} & \multirow[b]{2}{*}{$\begin{array}{l}\text { 体长 } \\
\text { Body length }\end{array}$} & \multirow[b]{2}{*}{$\begin{array}{l}\text { 翅长 } \\
\text { Wing length }\end{array}$} & \multirow[b]{2}{*}{$\begin{array}{l}\text { 跗蹠 } \\
\text { Tarsus length }\end{array}$} & \multirow[b]{2}{*}{$\begin{array}{l}\text { 参考文献 } \\
\text { References }\end{array}$} \\
\hline & $\begin{array}{c}\text { 网捕 } \\
\text { Mist net }\end{array}$ & $\begin{array}{c}\text { 红外相机 } \\
\text { Camera } \\
\text { trapping }\end{array}$ & $\begin{array}{c}\text { 网捕 } \\
\text { Mist net }\end{array}$ & $\begin{array}{c}\text { 红外相机 } \\
\text { Camera } \\
\text { trapping }\end{array}$ & & & & & \\
\hline 白腹凤鹤 Erpornis zantholeuca & 4 & 0 & 3 & 0 & $10.02 \pm 0.32(30)$ & $110.26 \pm 1.35(37)$ & $63.41 \pm 0.55(37)$ & $17.67 \pm 0.52(36)$ & 本研究 \\
\hline 金色鸦雀 Paradoxornis verreauxi & 3 & 0 & & & $5.46 \pm 0.1(13)$ & $95.91 \pm 1.07(11)$ & $46.07 \pm 0.35(13)$ & $18 \pm 0.39(12)$ & 本研究 \\
\hline 黄腰柳荣 Phylloscopus proregulus & 4 & 0 & 2 & 0 & $5.01 \pm 0.2(5)$ & $94.88 \pm 1.6(37)$ & $52.24 \pm 0.67(37)$ & $17.23 \pm 0.31(37)$ & 本研究 \\
\hline 淡脚柳莺 P. tenellipes & & & 1 & 0 & $11.5 \pm 1.5(2)$ & $115.75 \pm 3.15(4)$ & $61 \pm 1.54(4)$ & $20.25 \pm 0.32(4)$ & 赵正阶, 2001b \\
\hline 极北柳莺 P. borealis & & & 1 & 0 & $7.2 \pm 0.7(2)$ & $110.77 \pm 5(22)$ & $63.77 \pm 0.78(22)$ & $20.73 \pm 0.32(22)$ & 本研究 \\
\hline 栗头暡莺 Seicercus castaniceps & 2 & 0 & & & $5.3 \pm 0.24(4)$ & $89 \pm 1.18(5)$ & $50.2 \pm 1.07(5)$ & $13.65 \pm 1.54(4)$ & 本研究 \\
\hline 白眶叙莺 S. affinis & 2 & 0 & & & $6.96 \pm 0.1(9)$ & $104.85 \pm 14.43(9)$ & $59.64 \pm 1.2(9)$ & $20.66 \pm 0.33(9)$ & 本研究 \\
\hline 比氏鹟莺 S. valentini & 8 & 0 & & & $7.45 \pm 0.05(2)$ & $109.33 \pm 1.76(3)$ & $55 \pm 0.58(3)$ & $19 \pm 0(3)$ & 本研究 \\
\hline 叉尾太阳鸟 Aethopyga christinae & & & 1 & 0 & $5.91 \pm 0.25(4)$ & $105.19 \pm 3.61(13)$ & $49 \pm 0.68(13)$ & $15.03 \pm 0.37(10)$ & 本研究 \\
\hline 黄颊山雀 Parus spilonotus & 9 & 8 & & & $15.65 \pm 0.74(14)$ & $128.88 \pm 2.05(17)$ & $75.56 \pm 0.81(17)$ & $20.25 \pm 0.89(17)$ & 本研究 \\
\hline 白眉鳿 Emberiza tristrami & 0 & 1 & 7 & 0 & $14.92 \pm 1.14(2)$ & $135.09 \pm 1.57(47)$ & $70.43 \pm 0.48(47)$ & $20.42 \pm 0.24(43)$ & 本研究 \\
\hline 黄眉鴊 E. chrysophrys & & & 8 & 0 & $19.75 \pm 2.24(4)$ & $141.49 \pm 1.49(12)$ & $76.3 \pm 1.18(12)$ & $21.27 \pm 0.27(10)$ & 本研究 \\
\hline
\end{tabular}

$\uparrow:$ The handboook of the birds of the worlds, http://www.hbw.com/ 